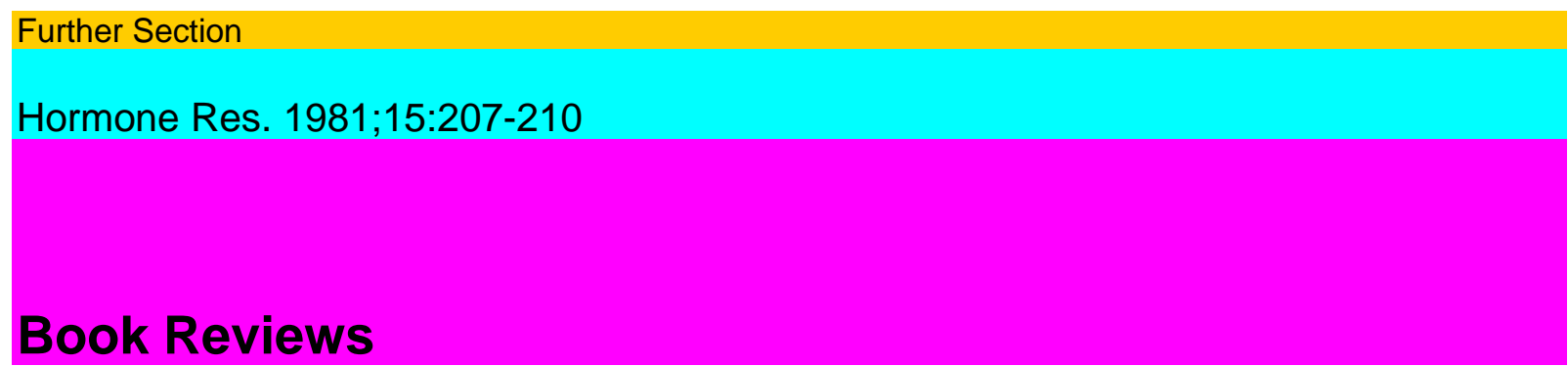

\title{
M. Beato
}

Steroid-Induced Uterine Proteins

Developments in Endocrinology, vol. 8 Elsevier/North-Holland, Amsterdam 1980 X + 380 pp.; US \$ 56.00 I Dfl. 115.-ISBN 0-444-80203-7

This book gives a rather well-documented review of one of the most exciting new aspects of hormone action: the induction of specific proteins by steroid hormones. The chapters on receptors demonstrate that uterine marker proteins may become convenient markers for the mechanism of estrogen action and the determination of estrogen responsiveness in both research and clinical application.

T.B. Schwartz, W.G. Ryan (eds.)

The 1980 Year Book of Endocrinology

Year Book Medical Publishers, London 1980384 pp.; US \$ 37.50

The present issue of this series presents again a most informative cross section through the recent reports of human and animal endocrinology. The topics discussed include quotations stretching from neuroendocrine surgery to endorphins, from the soma-tostatinoma syndrome to estrogen therapy of tall girls. Last but not least, the editors ask the reader to greet a new chapter on geriatric endocrinology. The book is well written and a pleasure to read.

W.B. Essman, (ed.)

Perspectives in Clinical Endocrinology

MTP Press, Lancaster 1981425 pp.; E 23.50 ISBN 0-85200-554-7

The book gives an expert review on selected topics of theoretical as well as clinical interest. The more recent literature is reviewed and a variety of aspects of central regulation of endocrine function are dealt with in the light of personal experience. The first chapter is devoted to the actual knowledge on neurotransmitter control of pituitary function. Very much space is given to the possible neuroendocrine effect of drugs acting on the central

Book Reviews

208

nervous system, whereas the pharmacological regulation of gonadotropin and prolactin secretion is treated in a more concise manner. Four chapters on gynecological aspects start off with pharmacodynamics in gynecological endocrinology and give very practical guidelines to thyroid and parathyroid dysfunction during pregnancy, as well as practical and theoretical aspects of diabetes mellitus in pregnancy. A further chapter is devoted to growth hormone dynamics in diabetes mellitus and another one to the recent literature on medullary thyroid carcinoma. A final chapter covers hormones of the gastrointestinal system which close the loop to the hormones of the central nervous system. Although the theoretical or practical aspects are unevenly distributed among the different topics, the Perspectives in Clinical Endocrinology are well selected, easy to read, and give a very comprehensive and up-to-date review on the subjects treated, providing a large list of references. An index gives access to specific questions.

F. Belfiore 
Enzyme Regulation and Metabolic Diseases

Karger, Basel 1980

XXIV + 880 pp.; SFr. 249.-

ISBN 3-8055-0005-X

This single-author monograph represents an almost gigantic review of enzyme alterations associated with the most important metabolic disorders. The author, Dr. F. Belfiore, made an enormous effort to embed in this review his own work in the area of enzyme regulation which started more than 15 years ago. The first part of the book is devoted to obesity, and summarizes regulatory enzymes of metabolic pathways which are altered in obesity, including enzymes of fatty acid and triglyceride metabolism. Part 2 covers the area of diabetes mellitus; it reviews the alterations of regulatory enzymes of metabolic pathways most frequently involved in diabetes. Particularly valuable is a review of enzymes of ketone body metabolism, and a general review of the in vitro work of enzymatic and metabolic abnormalities present in diabetes mellitus. This part will probably find widespread attention since it touches an area in which there is currently a high research activity. Part 3 is devoted to the subject of hyperlipoproteinemias, including general concepts of the subject, enzyme changes in hypertriglyceridemias and in hypercholesterolemias. The fourth part is entitled 'Atherosclerosis and Diabetic Microangiopathy'. It includes chapters on regulatory enzymes of the arterial wall, and on enzyme changes in atherosclerosis and diabetic microangiopathy. The text part of the book ends after 539 pages, and the remaining 338 pages include an impressive reference list of 4,391 titles. Nevertheless, the most recent work might not have been considered since all references are dated 1978 or earlier.

The book resembles an encyclopedia of the subject of enzyme regulation, and it is strongly recommended as a reference work for scientists working in the area of biochemistry of diabetes mellitus, or for all those interested in metabolic aspects of endocrinology, aging, hyperlipidemias and nutrition.

U. Keller, Basel

Book Reviews

209

A.J. Thody

The MSH Peptides

Academic Press, New York 1980 VIII + 162 pp.; US \$ 38.50/E 16.00 ISBN 0-12-687850-1

Prof. Thody has written an excellent readable account of the melanotrophin (MSH) peptides. By restricting the survey to mammals where most of the recent advances in this field have been made and by selecting data which contribute mainly to the physiological aspects of MSH, he has been able to condense the material into a handy-sized volume.

The book is divided into 9 chapters. The first three deal with the localisation of MSH peptides in pars intermedia and brain and their assay. In the following sections the secretion of pituitary MSH, MSH pigmentation and other peripheral effects, the effects of MSH on behaviour and a possible function for alpha-MSH in the fetal and neonatal animal are discussed. The final chapter covers the significance of MSH in the mammal, an, as yet, unsolved problem. The literature of over 600 references covers the topic up to 1980.

This volume will be of considerable value to all interested in the role of MSH and related peptides in mammals.

J.B. Baumann, Basel

M. Briggs, A. Corbin

Progress in Hormone Biochemistry and Pharmacology, Vol. 1 
MTP Press, Lancaster 1980

301 pp.;E 15.95

ISBN 0-85200-547^1

This book is the first volume of a new periodical summarizing recent progress in hormone biochemistry and pharmacology. It contains four chapters which review the current literature of an area in which recently discovered hormones or related compounds have stimulated widespread research activity. The editor's intention was to identify workers in such rapidly growing areas of endocrine biochemistry and pharmacology, and to invite them to prepare a definitive presentation of their field. By using camera-ready manuscripts, the volume was produced and released within 3 months. This in turn carried the disadvantage that the letter size of the printing is too small (less than $2 \mathrm{~mm}$ ) so that reading is sometimes tiresome.

Chapter 1 is devoted to the renin-angiotensin system, converting enzyme inhibition, and to antihypertensive therapy, written by B. Rubin et al., 54 pages cover the most recent work on the biochemical and clinical effects and side effects of converting enzyme inhibitors.

Chapter 2 is written by J.E. Gerich, an expert in the field of pharmacology and potential clinical application of somatostatin and its analogues. It represents a carefully written and invaluable update review of this rapidly developing area. As the title indicates, the author maintains the contention that such compounds are promising agents in the

Book Reviews

210

treatment of human disorders, such as diabetes mellitus. The chapter covers all the most relevant references (more than 300) of the subject.

Chapter 3 is entitled 'Botanical sources of fertility-regulating agents. Chemistry and pharmacology' by A.S. Bingel et al. It summarizes mainly work on animal fertility regulation agents from botanical sources. This chapter is a valuable source of references for scientists working in the area of fertility control; it should stimulate new research in the field of contraceptives but its topics are not devoted to clinical management of control of fertility. The last section is devoted to the luteinizing hormone-releasing hormone (LHRH) and analogues, and to their conceptive and contraconceptive potential. It reviews the recent literature on LHRH and related peptides which are promising agents as 'physiological' compounds in the control of contraception and in the stimulation of ovulation in man. The major work in this area has been reported in animals, but the first clinical trials appear to be promising. A problem not yet solved is the fact that peptide hormones such as LHRH and analogues are not suitable for oral use.

The book is particularly recommended to scientists working in the specific areas addressed in the topics of the 4 chapters.

P.H. Sönksen and C. Lowy

Endocrine and Metabolic Emergencies

Clinics in Endocrinology and Metabolism, vol. 9, No. 3 Saunders, Eastbourne 1980 VI + 643

pp.; E 9.75 ISBN 0-7216-8502-1

This volume reviews the more common endocrine and metabolic emergencies with which hospital doctors in particular and also practitioners will be faced.

Besides emergencies of specific glands, various chapters consider the disorders of acid-base balance, lactic acidosis and disturbances of electrolyte homeostasis. Special chapters are devoted to metabolic and endocrine emergencies in pregnancy, in the perinatal period as well as in psychiatric practice. 
The book is very readable and clearly presented - some chapters are illustrated with case histories - and can be recommended as a practical guide for diagnosis and treatment.

W. Ruch, Basel 